\title{
Pilot study on burden of fungal contamination in face masks: need for better mask hygiene in the COVID-19 era
}

\author{
Vishakh C Keri' ${ }^{1}$ Arvind Kumar¹, Gagandeep Singh², Ayan Mandal' ${ }^{1}$, Haider Ali', \\ Piyush Ranjan', Naveet Wig'1 \\ ${ }^{1}$ Department of Medicine, All India Institute of Medical Sciences, New Delhi, India; \\ ${ }^{2}$ Department of Microbiology, All India Institute of Medical Sciences, New Delhi, India
}

Article received 10 October, 2021; accepted 20 November, 2021

\section{SUMMARY}

Risk factors which led to the outbreak of COVID-19 associated Mucormycosis still remains elusive. Face masks can become contaminated by fungal spores that are present ubiquitously in the environment. However the exact burden of such contamination is not known. Fifty masks of patients who attended the Employees Health Scheme COVID-19 facility of a tertiary healthcare centre in India were sampled by direct impression smears on Sabouraud Dextrose Agar. Agar plates were screened for any growth within five days after incubation. Growth was identified by microscopy on the Lactophenol Cotton Blue mount. Mask hygiene practices of participants was assessed using a pre-designed proforma.

Out of 50 masks, fungal contamination was seen in 35/50 (70\%) masks, with Aspergillus sp. being isolated from 26/50 (52\%) masks and Mucorales being isolated from $9 / 50(18 \%)$ of the masks. Aspergillus niger, Rhizopus arrhizus and Syncephalastrum sp. were the most common species isolated. Same mask was worn for a median duration of 8 days (2-30 days) at a stretch with or without washing. Thirty one patients washed and re-wore their masks, with median time duration since last wash being 12 hours (4-72 hours). None of the factors assessed for mask hygiene were associated with fungal contamination.

High rates of fungal contamination observed in our study emphasizes the need for better mask hygiene in the COVID-19 era.

Keywords: COVID-19, Mucormycosis, facemask, mask hygiene.

\section{INTRODUCTION}

W earing face mask, social distancing and vaccination represents the core preventive strategies to halt the spread of COVID-19. Strict governmental rules have been laid down to ensure face masks are consistently used. A variety of face masks including N95, surgical and cloth masks are in use by general population. There are recommendations made by competent authorities

Corresponding author

Arvind Kumar

E-mail: linktoarvind@gmail.com for reuse of these masks [1]. Potential contamination of reused masks by fungal and bacterial pathogens is a matter of serious concern unless proper mask hygiene is maintained. The possible factors which led to a surge in cases of mucormycosis during COVID-19 pandemic are still unclear. Rapid increase in COVID-19 associated mucormycosis (CAM) challenged the healthcare system of India which was battling the second wave of COVID-19 pandemic. By the end of June 2021, India reported over forty thousand cases of mucormycosis and $85.5 \%$ of them were affected by COVID-19 [2]. Risk factors associated with a surge in mucor cases among patients with COVID-19 
is still elusive. Excessive use of steroids coupled with poor glycaemic control have been attributed as the cause for increase in CAM [3]. COVID-19 induced immune dysregulation, genetic susceptibility of Indian population, enhanced virulence of the prevalent Rhizopus arrhizus or the new delta variant increasing the susceptibility to Mucorales are some of the unresolved clinical questions [4]. Spores of mucorales are prevalent ubiquitously in the environment [5]. They are present in dead and decaying organic matter. Interestingly, hospital outbreaks have occurred due to contaminated linen, bandages in burns patients, wooden tongue depressors and stoma bags [6-8]. Thus environmental contamination of inanimate objects can act as a potential source of infection. Masking is a norm to prevent the spread of COVID-19. However similar environmental contamination of unwashed masks is possible and may act as a source of mucormycosis. Through this study we aimed to look at fungal contamination of face masks and estimate the burden of contamination by Mucorales. We also assessed association between fungal contamination and various determinants of mask hygiene among general population. Till date there are no studies which have tried to ascertain this fact.

\section{MATERIALS AND METHODS}

A cross sectional study was conducted over a period of two months (May and June 2021) among patients visiting the Employees Health Scheme (EHS) out-patient department (OPD) COVID-19 facility of All India Institute of Medical Science, New Delhi. As this was a pilot-study we planned to sample face masks of 50 individuals to look for fungal contamination. This study was undertaken after receiving ethical approval from institute ethics committee.

Samples from face masks were taken as direct impression smears on Sabouraud Dextrose Agar (SDA). Both the inner and outer surface of the mask were sampled. Agar plates were incubated at 38 degree Celsius for a maximum of five days to look for any growth. The growth was identified by colony characteristics and by microscopy based on morphology on Lactophenol Cotton Blue (LPCB) mount.

We also collected information about their demographic characteristics and mask hygiene practic- es in a predesigned form. Demographic characteristics included age, sex, type of mask worn. Mask hygiene practice including frequency of washing the cloth mask and regularity in changing the mask was assessed.

All statistical analysis was done using SPSS ver.23. Descriptive analysis was done to assess the nature of fungal contamination on face masks. Data for categorical variables was presented as frequency and percentages and continuous data was presented as mean \pm standard deviation or median/ IQR. Association between mask hygiene and fungal contamination of face mask was assessed using Chi square/Fischer exact test or Kruskal Wallis $\mathrm{H}$ test as appropriate. A p-value of less than 0.05 was considered significant for all tests.

\section{RESULTS}

Fifty masks were sampled from patients who visited EHS Screening OPD between 28 May 2021

Table 1 - Demographics, parameters of mask hygiene and details of fungal isolation.

\begin{tabular}{|c|c|}
\hline Parameters & $\begin{array}{c}n(\%) \\
\text { or Median (IQR) }\end{array}$ \\
\hline \multicolumn{2}{|l|}{ DEMOGRAPHIC DATA $(N=50)$} \\
\hline Age (years) & $36(25-49)$ \\
\hline Male & $33(66)$ \\
\hline \multicolumn{2}{|l|}{ MASK TYPE DETAILS $(N=48)$} \\
\hline N95 & $8(16.7)$ \\
\hline Surgical & $12(25)$ \\
\hline Cloth & $28(58.3)$ \\
\hline \multicolumn{2}{|l|}{ MASK HYGIENE DETAILS $(N=48)$} \\
\hline Duration of wearing same mask (days) & $8(2-30)$ \\
\hline Mask washed & $31(64.6)$ \\
\hline Time since last wash (hours) & $12(4-72)$ \\
\hline \multicolumn{2}{|l|}{ FUNGAL GROWTH $(N=50)$} \\
\hline No growth & $15(30)$ \\
\hline Aspergillus sp. & $26(52)$ \\
\hline Mucorsp. & $9(18)$ \\
\hline \multicolumn{2}{|l|}{ FUNGAL GROWTH INSIDE $(N=21)$} \\
\hline No growth & $7(33.3)$ \\
\hline Aspergillus sp. & $14(66.7)$ \\
\hline
\end{tabular}

Footnote: " $\mathrm{N}$ " indicates the total number of samples for which the data was available. 
and 28 June 2021. The median age of patients was 36 years (25-49 years) and $33(66 \%)$ were males. Cloth mask was used by $28(58.3 \%)$ patients, 12 (25\%) patients used surgical masks and $8(16.7 \%)$ of them used N95 masks. Individuals changed their masks after wearing the same mask for a median duration of 8 days (2-30 days) with or without washing. Thirty one patients washed and re-wore their masks, with median time duration since last wash being 12 hours (4-72 hours) (Table 1).

Out of 50 masks, fungal contamination was seen in 35/50 (70\%) masks, with Aspergillus sp. being isolated from 26/50 (52\%) masks. Aspergillus ni-

Table 2 - Detailed breakup of fungal contamination in masks (by species).

\begin{tabular}{|l|c|c|}
\hline \multicolumn{1}{|c|}{ Species } & $\begin{array}{c}\text { Outside layer } \\
(\mathrm{N}=50)\end{array}$ & $\begin{array}{c}\text { Inside layer } \\
(\mathrm{N}=21)\end{array}$ \\
\hline Mucor sp. & 2 & \\
\hline Rhizomucor sp. & 1 & \\
\hline Rhizopus arrhizus & 3 & \\
\hline Syncephalastrum sp. & 3 & \\
\hline Aspergillus flavus & 1 & 3 \\
\hline Aspergillus niger & 1 & 7 \\
\hline Aspergillus fumigatus & 1 & \\
\hline A. flavus + A. fumigatus & 8 & 2 \\
\hline A. niger + A. flavus & 1 & \\
\hline A. niger + A. fumigatus & 3 & \\
\hline $\begin{array}{l}\text { A. niger }+ \text { A. flavus } \\
+ \text { A. fumigatus }\end{array}$ & 32 & $\mathbf{1 4}$ \\
\hline Total & & \\
\hline
\end{tabular}

Footnote: " $\mathrm{N}$ " indicates the total number of samples for which the data was available. ger was the dominant subspecies, isolated from 8 (16\%) masks. Multiple Aspergillus species were also isolated from the same mask in 13/26 (50\%) masks. Mucor species were isolated in 9/50 (18\%) masks; Rhizopus arrhizus and Syncephalastrum were the most common species isolated (Table 2). Fungal contamination in the inner layer of 21 masks was also studied separately. Aspergillus sp. was isolated in 14/21 (66.7\%) masks however no Mucor sp. could be isolated.

No association was found between type of fungal isolate and either age $(p=0.67)$ or gender $(\mathrm{p}=0.62)$. A significant association was also not found between the type of fungal isolate and parameters for mask hygiene (mask washing, $\mathrm{p}=0.16$; duration of wearing same mask, $\mathrm{p}=0.33$; and time since last wash, $\mathrm{p}=0.11$ ). The type of mask used by the patients also did not show any significant association with fungal contamination $(\mathrm{p}=0.41)$ (Table 3).

\section{DISCUSSION}

With compulsory masking, mask hygiene has become a topic of great interest especially after the outbreak of mucormycosis. Although multiple theories have been suggested to explain the outbreak, the actual reason still remains unknown. Our study looked at the degree of contamination of face masks worn by general public with special emphasis at fungal contamination. We could demonstrate an astonishingly high percentage $(70 \%)$ of fungal contamination of face masks. While Aspergillus sp. was the predominant isolate, we were also able to isolate Mucorales in $18 \%$ of the masks. Fungal isolation was seen in both inner and outer surface of the mask how-

Table 3 - Association between demographics, mask hygiene with fungal contamination.

\begin{tabular}{|l|c|c|c|c|}
\hline \multicolumn{1}{|c|}{ Parameters } & No growth & Aspergillus sp. & Mucor sp. & p-value \\
\hline Male, $\mathrm{n}(\%)$ & $11(22)$ & $17(34)$ & $5(10)$ & 0.62 \\
\hline N95 mask (N=8) n (\%) & $1(12.5)$ & $5(62.5)$ & $2(25)$ & 0.41 \\
\hline Surgical mask (N=12) n (\%) & $3(25)$ & $8(66.7)$ & $1(8.3)$ & \\
\hline Cloth mask (N=28) $\mathrm{n}(\%)$ & $11(39.3)$ & $11(39.3)$ & $6(21.4)$ & 0.16 \\
\hline Mask washed (N=31) $\mathrm{n}(\%)$ & $10(20.8)$ & $13(27.1)$ & $8(16.7)$ & 0.67 \\
\hline Age (N=50) & $30(18-53)$ & $36(26-46)$ & $35(20-49)$ & 0.33 \\
\hline Duration of wearing same mask (days) (N=48) & $13(3-30)$ & $6(2-14)$ & $8(4-45)$ & 0.11 \\
\hline Time since last wash (hours) (N=48) & $6(3-72)$ & $36(5-72)$ & $4(2-24)$ & \multicolumn{2}{|c|}{0} \\
\hline
\end{tabular}


ever we were not able to find any Mucorales on the inner side. Studies among health care workers showed that $1.9 \%-28.3 \%$ unwashed aprons were contaminated by fungal species which also included species of Mucorales [9, 10]. However, studies investigating face mask contamination are not available.

Fungal spores are ubiquitous in the environment and are known to contaminate inanimate objects, and have also been implicated in hospital outbreaks $[6,11]$. However the high burden of fungal contamination might reflect poor mask hygiene. Isolation of Mucorales also shows that masks could be a possible source for mucormycosis in the absence of adequate mask hygiene among reused masks. Prolonged use of cloth and surgical masks were associated with higher odds (aOR 6.9, 95\%CI 1.5-33.1) of developing CAM in a recent study from Northern India [12].

In our study cloth masks were most commonly used. They were used for a median duration of 8 days at a stretch and were washed after a median duration of 12 hours. Although in our study we were not able to demonstrate association between factors of mask hygiene and fungal contamination due to low sample size, we urge that the general population continue to adhere to maintaining good mask hygiene in view of high fungal burden that we observed in the sampled masks. World Health Organisation recommends that non-medical fabric masks be washed at-least once a day with soap or detergent, preferably with hot water [13].

This pilot study serves as a primer for further studies to look for fungal contamination of face masks. It also emphasizes the need to look into other factors responsible for poor mask hygiene apart from the obvious ones discussed in our study. It will act as a good opportunity to educate and sensitize general population on proper mask hygiene. More targeted evidence based guidelines on reuse of mask and mask hygiene are required. Mere isolation of fungal species from masks may not translate into developing infection. More follow up studies to look for factors which predispose persons with fungal contamination to develop infection may be required to ascertain causality. Face masks have become an important part of our lives now owing to the COVID-19 pandemic, and this study stresses on the importance of need for better mask hygiene.

\section{CONCLUSION}

High rates of fungal contamination of face masks in our study impresses upon the need for better mask hygiene. Guidelines on use, reuse and decontamination of face masks is the need of the hour.

\section{Conflict of interest}

Authors do not have any conflict of interest to declare.

\section{Funding}

None.

\section{Ethical clearance}

Obtained.

\section{REFERENCES}

[1] Toomey EC, Conway Y, Burton C, et al. Extended use or reuse of single-use surgical masks and filtering face-piece respirators during the coronavirus disease 2019 (COVID-19) pandemic: A rapid systematic review. Infect Control Hosp Epidemiol. 2021; 42, 75-83.

[2] Chandra NS. India reports 40,854 cases of black fungus so far. https://www.livemint.com/news/india-records-over-40k-cases-of-mucormycosis-11624875874985. html.

[3] Sen M, Honavar SG, Bansal R, et al. Epidemiology, clinical profile, management, and outcome of COVID-19-associated rhino-orbital-cerebral mucormycosis in 2826 patients in India - Collaborative OPAI-IJO Study on Mucormycosis in COVID-19 (COSMIC), Report 1. Indian J Ophthalmol. 69, https://journals.lww. com/ijo/Fulltext/2021/07000/Epidemiology,_clinical_profile,_management,_and.10.aspx (2021).

[4] Chakrabarti A. The recent mucormycosis storm over Indian sky. Indian J Med Microbiol. 2021; S02550857(21)04134-7.

[5] Prakash H, Chakrabarti A. Global Epidemiology of Mucormycosis. J Fungi (Basel) 2019; 5, 26.

[6] Cheng VCC, Chen JHK, Wong SCY, et al. Hospital outbreak of pulmonary and cutaneous Zygomycosis due to contaminated linen items from substandard laundry. Clin Infect Dis. 2016; 62, 714-721.

[7] Rammaert B, Lanternier F, Zahar J-R, et al. Healthcare-Associated Mucormycosis. Clin Infect Dis. 2012; 54, S44-S54.

[8] Devauchelle P, Jeanne M, Fréalle E. Mucormycosis in Burn Patients. J Fungi (Basel). 2019; 5: 25.

[9] Keri VC, Kumar A, Singh G, et al. Fungal carriage on healthcare workers' hands, clothing, stethoscopes and electronic devices during routine patient care: a study 
from a tertiary care center. J Prev Med Hyg; 62. Epub ahead of print 29 April 2021. DOI: $10.15167 / 2421-4248 /$ jpmh2021.62.1.1645.

[10] Shermil S, VP S, Shamsudeen S, et al. Identification of microbial isolations from the clinical aprons of graduates and post-graduate trainees in a college department. Acta Scientific Dental Sciences. 2018; 8-15.

[11] Duffy J, Harris J, Gade L, et al. Mucormycosis Outbreak Associated with Hospital Linens. Ped Infect Dis J. 33, https:/ /journals.lww.com/pidj/Fulltext/2014/05000/
Mucormycosis_Outbreak_Associated_With_Hospital.9.aspx (2014).

[12] Arora U, Priyadarshi M, Katiyar V, et al. Novel risk factors for Coronavirus disease-associated mucormycosis (CAM): a case control study during the outbreak in India. medRxiv. 2021; 2021.07.24.21261040.

[13] How to wear a non-medical fabric mask safely, https: / / www.who.int/images/default-source/healthtopics / coronavirus / clothing-masks-infographic--(web)-logo-who.png?sfvrsn=b15e3742_18. 